\title{
Research on Capital Asset and Investment Portfolio
}

$$
\text { Zeyu } \mathrm{Li}^{1, \mathrm{a},{ }^{*}, \dagger}{ }^{\dagger} \mathrm{Jin} \mathrm{Liu}^{2, \mathrm{~b},{ }^{*},{ }^{\dagger}} \text { Yixin Zhang }{ }^{3, \mathrm{c}, *,{ }^{*}} \text { Yuqian } \mathrm{Zou}^{4, *},{ }^{,},{ }^{\dagger}
$$

\author{
${ }^{1}$ International Business Faculty, Beijing Normal University Zhuhai, Zhuhai, 519087, China \\ ${ }^{2}$ Department of Psychology, University of Washington, Seattle, 98105, U.S. \\ ${ }^{3}$ HKU Business school, The University of Hong Kong, Hong Kong, China \\ ${ }^{4}$ School of Social Science Business Economics, University of California, Irvine, Irvine,92617, U.S. \\ *Corresonding author.Email: ${ }^{a}$ tangdaren301@gmail.com ${ }^{b}$ jliu4@uw.edu ${ }^{c}$ zyx0508@ connect.hku.hk, \\ dyuqianz5@uci.edu These authors contribute equally.
}

\begin{abstract}
Throughout history, using capital assets models, such as Markowitz and Index model, is important for investors to compare different portfolios, and decide the asset arrangement. Doing statistical analysis and comparisons of stock prices for different companies is necessary when we want to find the optimal portfolio to make an investment. This research analyzed six corporations' stock indexes from 2000 to 2020 by using the Markowitz model and Index model. Besides, five different constraints are put and discussed to make the research more useful to implement in realistic settings. The results calculated using the Markowitz and Index model are similar, even though there are slight differences under different constraints. The real meaning of our research is that using the Markowitz model leads to higher returns for the portfolio investment when both models are set to have a maximum standard deviation. Our research provides empirical insight into the investor's decision.
\end{abstract}

Keywords: Index model, Markowitz model, Investment Portfolio, statistical constraints

\section{INTRODUCTION}

When thinking about investment portfolios, this can be defined as a collection of financial assets bought by investors to meet their financial goals. Examples of collection assets are funds, stocks, bonds, etc [1]. Throughout history, in 1938, John Burr Williams suggests that the stock value should equal its future dividends, price in his book - The theory of investment value. In 1952, Harry Markowitz developed the "modern portfolio theory" . The Markowitz model is a method that can match investors' risk tolerances with payoff expectations [2]. By putting this relationship on a graph, it shows that an ideal portfolio used to diversify asset investment. The development of modern portfolios was evolved by many economists adjust and contribute to different investment theories.

Using mathematical and statistical analysis to evaluate the value of a stock is significant. The existing theory about evaluating the optimal portfolio include the Markowitz model and Index model. However, the investment efficiency and value evaluation of these two models are different in the real market. This article considers lots of stocks to establish the investment portfolio. Besides, based on the minimum standard deviation and maximum Sharpe ratio, different sets of investment portfolios are created in order to capture the best investment portfolio models in the real market.

In the theoretical finance area, both Markowitz' $s$ model and index model have long been the pillars and mainstream theories. Proposed by Harry Markowitz in the 1950s, the Markowitz model identifies the relationship between risk and expected return [3]. For any given level of standard deviation, the model assumes that people would demand the highest return available. In this way, Markowitz helps to generate the efficient frontier and optimize the investment portfolio construction. Eventually, this theory becomes the foundation of wealth management and investment practice.

Based on the research of Harry Markowitz, William Sharpe developed the index model by adding more assumptions to simplify Markowitz theory. As Kamal [4] stated, Sharpe' $\mathrm{s}$ index model assumed that the comovements between different stocks are derived from the variation of the stock index. In addition, the risk of any asset can be divided into systematic risk and non- 
systematic risk. The model aims at finding the optimal asset by considering the expected return as well as the systematic risk (i.e. $\beta$ ) minimizing the standard deviation when calculate and maximizing the Sharpe ratio. Five different constraints are added to each model and with the two objectives mentions above. The data of Standard \& Poor's 500 Index (SPX) and six companies are used, which are Adobe, IBM (International Business Machines) Corporation, Bank of America, Citigroup, Southwest Airlines, and Alaska Airlines. During the whole project, Excel and the solver table are used to compute the scenarios of maximum returns, maximum Sharpe Ratio, and work out the Efficient Frontiers, Minimal Returns Frontiers, and the graph of each outcome. Compare between the two specific models, when the returns are equal, Markowitz Model generally generates a slightly higher variance than the one computed by the Index Model. This indicated that the Markowitz Model has a return with a higher risk and a higher Sharpe ratio than Index Model. Moreover, when the different constraints being added, Markowitz will give higher returns with a higher risk and a higher Sharpe ratio. Since there is a negative relationship between the returns and risks, also, the returns and Sharpe ratios.

The remainder of the paper is organized as follows: Section 2 describes the data and the company; Section 3 conducts the constraints of the assumptions and the Markowitz Model and Index model. Section 4 introduces two indicators used to measure the portfolio called minimal standard deviation and max sharp ratio. Section 5 performs the result analysis of minimal variance and max sharp ratio. The last section presents our conclusions.

\section{DATA}

20 years of total return data for six stocks in history are used to demonstrate the practical usage of this research and these companies come from three different industry groups. The input takes daily returns from the Standard and Poor's 500 (S\&P 500) and six large corporations' stock indexes from 2000 to 2020. The S\&P 500 index measures the "market capitalizationweighted index" of the 500 largest public trading companies in the U.S. Companies, market capitalizations, expressed as a number of stocks traded publicly can vary based on this float-weighted index [5]. Another rate used is a proxy for the risk-free rate and a 1month annual Fed Funds rate is put into the calculation. The Federal Open Market Committee set the risk-free rate to charge commercial banks who borrow and lend their excess returns between each other overnight [6]. Next, six stocks come from companies: Adobe, IBM, Bank of America, Citigroup, Southwest Airlines, and Alaska Airlines.
Adobe is a software company that aims to provide creative, document, and marketing software to help customers manage their content. IBM (International Business Machines) is a corporation that offers information technology products and services to customers. The company' s main segments are Cloud Software, Global Technology Services, Business Services, and Financing.

Citigroup is a company offering financial products and services. The specialists aim to deliver banking products and services to customers, like derivative services, foreign exchange, equity sales, and trading, etc.

Southwest Airlines is a low-cost airline carrier and the company covers domestic flights as well as international ones.

The last company is Alaska Airlines, which is a company that provides passenger airline services. Their main services include travel arrangers, reservations, baggage, pet travel, etc.

Besides, Table 1 shows the correlation relationship between six studied companies. Correlation means the product of correlations with the market index and all correlational relationships are decided by securities betas and market index properties. To calculate the correlational link, excess return should be measured first, which is the number of extra returns achieved by a stock than the risk-free rate. Based on the calculation of excess return, the results of the correlation are expressed in table 1. The correlation results measures levels of two variables move in relationship with each other. The values range from -1.0 to 1.0 and we assume that a company has a 1.0 correlation with itself, which is a perfectly positive correlation. The closer the correlation number to 1.0 indicates a stronger relationship that two companies vary together. The farther the correlation results from 1.0 shows the weaker relationship.

Next, Table 2 represents a series of descriptive statistics of seven companies and the input in calculations is their different monthly stock prices. The mean results calculate the average monthly stock prices for each company from 2000 to 2020 . The standard deviation measures the spread of a dataset from the mean number, which can indicate fluctuations in the stock prices. "Min" and "Max" show the smallest and largest numbers of the dataset. For example, considering the results of standard deviations of 6 companies, the company Citigroup has the largest standard deviation (207.9940) and Adobe has the lowest standard deviation (6.6433). Adobe has a lower standard deviation than Citigroup indicates that their stock prices tend to be closer to the mean number, and Citigroup' $s$ data set of stock prices spread in a larger values range. This means that Adobe' s stock prices have fewer fluctuations in changes than the Citigroup'sStatistics of the Correlational relationship between 7 companies. 
TABLE I. STATISTICS OF THE CORRELATIONAL RELATIONSHIP BETWEEN 7 COMPANIES

\begin{tabular}{|c|c|c|c|c|c|c|c|}
\hline \multirow{2}{*}{$\begin{array}{l}\text { Variable } \\
\text { SPX }\end{array}$} & & \multirow{2}{*}{$\begin{array}{c}\text { Mean } \\
2102.5060\end{array}$} & \multirow{2}{*}{$\begin{array}{l}\text { Std. dev. } \\
71.7418\end{array}$} & \multirow{2}{*}{$\begin{array}{c}\text { Min } \\
837.1229\end{array}$} & \multicolumn{2}{|r|}{ Max } & \\
\hline & & & & & \multicolumn{2}{|r|}{5255.7709} & \\
\hline ADBE & & 84.2778 & 6.6433 & 9.581 & \multicolumn{2}{|r|}{516.633} & \\
\hline IBM & & 153.8270 & 54.8014 & 58.9059 & \multicolumn{2}{|r|}{249.8343} & \\
\hline $\mathrm{BAC}$ & & 35.6616 & 15.9180 & 5.7508 & \multicolumn{2}{|r|}{69.1409} & \\
\hline$C$ & & 227.8762 & 207.9940 & 19.6304 & \multicolumn{2}{|r|}{659.3967} & \\
\hline LUV & & 25.6655 & 17.8449 & 5.9514 & \multicolumn{2}{|r|}{69.4549} & \\
\hline \multirow[t]{2}{*}{ ALK } & & 29.8751 & 28.9267 & 3.835 & \multicolumn{2}{|r|}{102.489} & \\
\hline & SPX & ADBE & IBM & BAC & C & LUV & ALK \\
\hline SPX & 1.00 & 0.64 & 0.64 & 0.59 & 0.70 & 0.53 & 0.46 \\
\hline ADBE & 0.64 & 1.00 & 0.40 & 0.39 & 0.44 & 0.37 & 0.21 \\
\hline $\mathrm{IBM}$ & 0.64 & 0.40 & 1.00 & 0.31 & 0.41 & 0.31 & 0.35 \\
\hline BAC & 0.59 & 0.39 & 0.31 & 1.00 & 0.82 & 0.40 & 0.25 \\
\hline C & 0.70 & 0.44 & 0.41 & 0.82 & 1.00 & 0.42 & 0.29 \\
\hline LUV & 0.53 & 0.37 & 0.31 & 0.40 & 0.42 & 1.00 & 0.49 \\
\hline ALK & 0.46 & 0.21 & 0.35 & 0.25 & 0.29 & 0.49 & 1.0 \\
\hline
\end{tabular}

\section{MethoD}

This research makes use of two different models to find the best asset allocation portfolio with respect to the above-presented data under five different constraints. The following part briefly introduces all the constraints, and then explain with clarity the basic arguments of the Markowitz model and index model.

\subsection{Five constraints}

The existence of five different constraints actually conveys some economic or financial information. Reallife conditions are simplified and five mathematical restrictions are used to simulate regulations that investors may face.

The first constraint gives that $\sum_{i=1}^{7}\left|w_{i}\right| \leq 2$, which is derived from the regulation $T$ of the FINRA (i.e. the financial industry regulatory authority). This regulation makes it clear that in general, firms can only lend up to $50 \%$ of the total purchase price of margin security, which means that the absolute value of any negative weighting of any securities cannot be larger than 0.5. in our case, once any individual asset has a value lower than negative 0.5 , given that the sum of all weighting is 1 , the total absolute value of the portfolio will exceed 2 . So the constraint shows congruence with the regulation $\mathrm{T}$. 
The second constraint is $\left|w_{i}\right| \leq 1$, for $\forall i$. This is a stimulation of box constraint, namely for a single value $\mathrm{x}$, a lower bound and an upper bound is set. In this specific case, the lower bound is a negative one whereas the upper bound is a positive one. This restriction serves as a proxy for some additional constraints that an investor may impose on the trading.

The third constraint actually imposes no additional restriction on portfolio construction. This scenario simulates the entirely free market without any limitation.

The fourth constraint regulates $w_{i} \geq 0$, for $\forall i$. In other words, no short position is allowed. Such limitation is typical in many markets. For instance, all the openended mutual fund in America is strictly prohibited from short selling.

The last constraint requires $w_{1}=0$, where $w_{1}$ represents the weighting of the Standard Poor index in the portfolio. Clearly, under this circumstance, the board index is excluded from the portfolio formation.

\subsection{Markowitz Model}

Harry Markowitz assumes that all investors are rational and risk-averse. In other words, investors will always try to minimize their risk at a given level of expected return or to maximize their return at a fixed level of risk. This model assumes that the return for an investment portfolio will be

$$
E\left(R_{p}\right)=\sum w_{i} \times E\left(R_{i}\right)
$$

where $\sigma_{i}$ is the variance of asset $\mathrm{i}$, and $\rho_{i j}$ describes the correlation coefficient between asset $i$ and asset $j$.

\subsection{Index Model}

Index model is another asset pricing model which suggests that the expected return of an asset should be proportional to its non-diversifiable risk (i.e. beta). Consequently, the index model treats market beta, rather than standard deviation, as a risk indicator. The return of an individual asset is described as

$$
R_{i}=\alpha_{i}+\beta_{i} \times R_{m}+e_{i}
$$

where $R_{m}$ represents market return and $e_{i}$ represents the residual value. In light of Sharpe's model, nonsystematic risk can be eliminated by diversification whereas systematic risk remains the only factor that leads to the volatility of asset price [7].

\section{Portfolio Investment}

In order to compare the MM and IM models and find the best asset allocation, a portfolio is built with the standard of minimal standard deviation and max sharp ratio under different constraints. Here are some descriptions of the two standards.

\subsection{Minimum Standard Deviation}

The term "portfolio variance" refers to a statistical value of modern investment theory that helps in the measurement of the dispersion of average returns of a portfolio from its mean. In short, it determines the total risk of the portfolio. It can be derived based on a weighted average of individual variance and mutual covariance. Mathematically, the portfolio variance formula consisting of two assets is represented as,

$$
\begin{gathered}
\mathrm{C}=w_{12} \times \mathrm{o}_{12}^{\prime}+w_{22} \times \mathrm{o}_{22}^{\prime}+2 \times \rho_{1}, 2 \times w_{1} \times \\
w_{2} \times \mathrm{o}_{1}^{\prime} \times \mathrm{o}_{2}^{\prime}
\end{gathered}
$$

where $w_{i}$ is Portfolio weight of asset $\mathrm{I}, o_{i}^{2}$ means individual variance of asset $\mathrm{I}, \rho_{i}, j$ is the correlation between asset I and asset j, C is Portfolio Variance Formula.

Again, the variance can be further extended to a portfolio of more no. of assets, for instance, a 3-asset portfolio can be represented as,

$$
\begin{gathered}
\mathrm{C}=\mathrm{w}_{12} \times \mathrm{o}_{12}+\mathrm{w}_{22} \times \mathrm{o}_{22}+\mathrm{w}_{32} \times \mathrm{o}_{32}+ \\
2 \times \rho_{1}, 2 \times \mathrm{w}_{1} \times \mathrm{w}_{2} \times \mathrm{o}_{1} \times \mathrm{o}_{2}+ \\
2 \times \rho_{2}, 3 \times \mathrm{w}_{2} \times \mathrm{w}_{3} \times \mathrm{o}_{2} \times \mathrm{o}_{3}+2 \times \rho_{3}, 1 \times \\
\mathrm{w}_{3} \times \mathrm{w}_{1} \times \mathrm{o}_{3} \times \mathrm{o}^{\prime}
\end{gathered}
$$

The portfolio variance formula of a particular portfolio can be derived by using the following steps:

(1) determine the weight of each asset in the overall portfolio, and it is calculated by dividing the asset value by the total value of the portfolio. The weight of the $i^{\text {th }}$ the asset is denoted by $w_{i}$.

(2) determine the standard deviation of each asset, and it is computed on the basis of the mean and actual return of each asset. The standard deviation of the $i^{\text {th }}$ the asset is denoted by $\sigma_{i}^{\prime}$. The square of the standard deviation is variance i. e. , $o_{i}^{2}$

(3) determine the correlation among the assets, and it basically captures the movement of each asset relative to another asset. The correlation is denoted by $\rho$

(4) the portfolio variance formula of two assets is derived based on a weighted average of individual variance and mutual covariance, as shown below.

$$
\begin{gathered}
\mathrm{C}=\mathrm{w}_{1} \times \mathrm{o}_{12}^{\prime}+\mathrm{w}_{2} \times \mathrm{o}_{22}^{\prime}+2 \times \rho_{1}, 2 \times \mathrm{w}_{1} \times \\
\mathrm{w}_{2} \times \mathrm{o}_{1}^{\prime} \times \mathrm{o}_{2}^{\prime}
\end{gathered}
$$

\subsection{Maximun Sharpe Ratio}

The ratio describes how much excess return investors receive for the extra volatility they endure for holding a 
riskier asset. Investors need compensation for the additional risk they take for not holding a risk-free asset. Sharpe Ratio is calculated by the following.

$$
\text { Sharpe Ratio }=\frac{\left(R_{x}-R_{f}\right)}{\sigma_{x}}
$$

Where: $R_{x}$ is Expected portfolio return, $R_{f}$ is Risk-free rate of return, $\sigma_{x}$ is Standard deviation of portfolio return (or, volatility)

The Sharpe ratio is a calculation of how much excess returns will be generated for each unit of total risk that the investment portfolio bears. The different Sharpe ratios generated by the MM model and the IM model are compared in five different scenarios to detect which model can better complete the asset allocation work.

\section{RESUlt ANALYSIS}

In this section, firstly, the objective cell is set as minimizing standard deviation, maximizing Sharpe ratio respectively to establish the different portfolios. Then the five constraints mentioned above are added one by one to each model. The different weights of each asset are compared with different constraints between the two models. Finally, the results are organized in Tables.

\subsection{Minimum Standard Deviation}

According to the above analysis, for the goal to minimizing the risk, represented by the standard deviation Table 3 and Table 4 are obtained.

TABLE II. MARKOWITZ MODEL RESULTS FOR MINIMUM STANDARD DEVIATION

\begin{tabular}{ccccccccccc}
\hline Constraint & SPX & ADBE & IBM & BAC & C & LUV & ALK & Returns & St. Dev & $\begin{array}{c}\text { Sharpe } \\
\text { Ratio }\end{array}$ \\
\hline$\sum_{i=1}^{7}\left|w_{i}\right| \leq 2$ & 1.35 & -0.13 & -0.02 & 0.06 & -0.23 & 0.01 & -0.05 & $6.52 \%$ & $13.10 \%$ & 0.50 \\
$\left|w_{i}\right| \leq 1$ & 1.00 & -0.06 & 0.13 & 0.09 & -0.21 & 0.07 & -0.01 & $7.27 \%$ & $13.78 \%$ & 0.53 \\
None & 1.35 & -0.13 & -0.02 & 0.06 & 0.23 & 0.01 & -0.05 & $6.52 \%$ & $13.10 \%$ & 0.50 \\
$w_{i} \geq 0$ & 1.00 & 0.00 & 0.00 & 0.00 & 0.00 & 0.00 & 0.00 & $6.16 \%$ & $15.10 \%$ & 0.41 \\
$w_{1}=0$ & 0.00 & 0.12 & 0.55 & 0.18 & -0.15 & 0.22 & 0.08 & $9.38 \%$ & $21.00 \%$ & 0.45 \\
\hline
\end{tabular}

TABLE III. INDEX MODEL RESULTS FOR MINIMUM STANDARD DEVIATION

\begin{tabular}{ccccccccccc}
\hline Constraint & SPX & ADBE & IBM & BAC & C & LUV & ALK & Returns & St. Dev & $\begin{array}{c}\text { Sharpe } \\
\text { Ratio }\end{array}$ \\
\hline$\sum_{i=1}^{7}\left|w_{i}\right| \leq 2$ & 1.43 & -0.11 & -0.02 & -0.09 & -0.17 & -0.02 & -0.02 & $5.94 \%$ & $12.92 \%$ & 0.46 \\
$\quad w_{i} \mid \leq 1$ & 1.00 & -0.04 & 0.14 & -0.05 & -0.14 & 0.06 & 0.03 & $6.79 \%$ & $13.84 \%$ & 0.49 \\
None & 1.43 & -0.11 & -0.02 & -0.09 & -0.17 & -0.02 & -0.02 & $5.94 \%$ & $5.94 \%$ & 0.46 \\
$w_{i} \geq 0$ & 1.00 & 0.00 & 0.00 & 0.00 & 0.00 & 0.00 & 0.00 & $6.16 \%$ & $15.10 \%$ & 0.41 \\
$w_{1}=0$ & 0.00 & 0.10 & 0.50 & 0.05 & -0.05 & 0.24 & 0.15 & $8.74 \%$ & $20.92 \%$ & 0.42 \\
\hline
\end{tabular}

The weights of assets in Table 3 are investigated for the Markowitz Model. When the first constraint $\sum_{i=1}^{7}\left|w_{i}\right| \leq 2$ being added, it shows that SPX has the weights of 1.35 , ADBE is allocated with -0.13 , IBM with -0.02 , BAC with 0.06 , Citigroup with -0.23 , LUV with 0.01 , ALK with -0.05 . Under this specific circumstance, a minimum standard deviation can be found. For the Index Model, the SPX has weights of 1.43, and 0.11, $0.02,-0.09,-0.17,-0.02$, and -0.02 respectively for the other six. Then the investment information of different portfolios is summarized based on two models in Table 3 and Table 4. Under the first constraint, $\sum \mid$ wi $\mid \leq 2$, Index Model has returns of $5.94 \%$ with a minimum standard deviation, $12.92 \%$. On the other hand, Markowitz Model has a return of $6.52 \%$, and a minimum standard deviation of $13.1 \%$. In the following constraint, Index Model has returns of $6.79 \%, 5.94 \%, 6.16 \%$, and $8.74 \%$, and respectively with a minimum standard deviation of $13.84 \%, 12.92 \%, 16.10 \%$, and $20.92 \%$. This vindicates 
the fact that the Markowitz Model overall produces a higher return with a higher risk.

\subsection{Maximum Sharpe Ratio}

Instead of minimizing the risk, the object is set as maximizing the Sharpe ratio with all five of the constraints adding each time. The results for the Markowitz Model is shown in Table 5, and Table 6 is presented with the outcomes of the Index Model.

TABLE IV. MARKOWITZ MODEL RESUlTS FOR MAXIMUM SHARPE RATIO

\begin{tabular}{cccccccccc}
\hline Constraint & ADBE & IBM & BAC & C & LUV & ALK & Returns & St. Dev & $\begin{array}{c}\text { Sharpe } \\
\text { Ratio }\end{array}$ \\
\hline$\sum_{i=1}^{7}\left|w_{i}\right| \leq 2$ & 0.21 & 0.00 & 0.3 & -0.5 & 0.00 & 0.18 & $15.12 \%$ & $18.40 \%$ & 0.82 \\
$\left|w_{i}\right| \leq 1$ & 0.29 & -0.16 & 0.50 & -0.76 & -0.1 & 0.25 & $19.43 \%$ & $22.61 \%$ & 0.86 \\
None & 0.29 & -0.16 & 0.50 & -0.76 & -0.1 & 0.25 & $19.43 \%$ & $22.61 \%$ & 0.86 \\
& & & & & & & & & \\
$w_{i} \geq 0$ & 0.56 & 0.00 & 0.00 & 0.00 & 0.00 & 0.44 & $16.13 \%$ & $27.76 \%$ & 0.58 \\
$w_{1}=0$ & 0.74 & 0.02 & 0.91 & -1.14 & -0.08 & 0.55 & $31.13 \%$ & $38.19 \%$ & 0.81 \\
\hline
\end{tabular}

TABLE V. INDEX MODEL RESULTS FOR MAXIMUM SHARPE RATIO

\begin{tabular}{ccccccccccc}
\hline Constraint & SPX & ADBE & IBM & BAC & C & LUV & ALK & Returns & St. Dev & $\begin{array}{c}\text { Sharpe } \\
\text { Ratio }\end{array}$ \\
\hline$\sum_{i=1}^{7}\left|w_{i}\right| \leq 2$ & 0.95 & 0.28 & -0.09 & 0.00 & -0.41 & 0.04 & 0.22 & $14.69 \%$ & $20.01 \%$ & 0.73 \\
$\left|w_{i}\right| \leq 1$ & 1.00 & 0.30 & -0.15 & 0.00 & -0.43 & 0.04 & 0.23 & $15.27 \%$ & $20.75 \%$ & 0.74 \\
None & 1.02 & 0.30 & -0.15 & 0.00 & -0.43 & 0.04 & 0.23 & $15.20 \%$ & $20.65 \%$ & 0.74 \\
$w_{i} \geq 0$ & 0.04 & 0.51 & 0.00 & 0.00 & 0.00 & 0.07 & 0.39 & $15.20 \%$ & $26.92 \%$ & 0.56 \\
$w_{1}=0$ & 0.00 & 0.72 & 0.00 & 0.14 & -0.57 & 0.21 & 0.51 & $23.80 \%$ & $34.52 \%$ & 0.69 \\
\hline
\end{tabular}

For the first constraint, SPX is assigned with weights of 0.89 , and $0.21,0,0.3,-0.5,0$, and 0.18 are assigned for each of the six companies. Markowitz Model has a maximum Sharpe ratio of 0.86 , with returns of $19.43 \%$. The Index Model makes a return of $14.69 \%$ with a maximum Sharpe ratio of 0.73. Basically, the Markowitz Model has higher returns and a higher Sharpe ratio. In the following constraints, the outcomes are similar as well, Index Model has lower returns with lower standard deviation and Sharpe ratio. The maximum returns value for Markowitz Model is $31.30 \%$ where the constraint is the fifth one, $w_{1}=0$, the inclusion of the broad index with the Sharpe ratio being maximized.

Regarding Index Model, the maximum return is $23.8 \%$ which is also under the constraint of $\mathrm{w} 1=0$, and with a maximum Sharpe ratio. The minimum returns of these two models happened when the risk is minimized and the first constraint, $\sum|w i| \leq 2$ is added. Markowitz has a return of $7.27 \%$, and the Index Model has a return of $5.94 \%$. So the constraint of including a broad index help generates higher returns for both models and the Regulation T by FINRA ("Under these rules, as a general matter, the customer's equity in the account must not fall below $25 \%$ of the current market value of the securities in the account") creates a strong limitation on the returns for both models [8].

After the calculation by using the solver, it can be perceived that the overall results calculated by the Markowitz Model and Index Model are similar since the Index Model is in fact, a simplification of the Markowitz model, and the two models basically come from the same source [9].

\section{Conclusion}

This research utilized the Markowitz model and index model to construct an optimal investment portfolio under five different constraints, all of which have specific economic and realistic meaning. Then, with each model and each constraint, the portfolio is generated with 
minimum variance, and the one with maximum Sharpe ratio. By yielding these two points, we aim at finding the optimal way of asset allocation.

It has been shown that the results of the two models are homogenous, and the rules of greater returns always with higher risk are shown in the whole process. However, if the two models are compared strictly, the Markowitz Model always creates a higher return with a higher standard deviation when the standard deviation is maximized. And it also has higher returns with a higher Sharpe ratio when maximizing the Sharpe ratio is set as the objective. This proves that the Markowitz model can be a little bit closer to the optimal solutions, with regards to the objective set. In addition to the conclusion from the model, we can also perceive that the influence being brought when each time a different constraint is being added. As a conclusion, the margin requirement of Regulation T by FINRA, limit the returns of each model with the most power. And the inclusion of a broad index will make the returns higher.

Three parts need us to improve in the follow-up research. Firstly, the different portfolio would affect the use of the models such as the industry correlation between companies in the portfolio. Besides is the use of the Markowitz Model and Index Model, these models ignore the effect of the investors' behaviors. The analysis of historical data also causes the problem of empiricism. The last one is the indicators. The implicit assumption of risk adjustment of returns by standard deviation is that the portfolio under review constitutes the whole of investor investment. So sharp ratios can only be an important basis when considering choosing to buy one of the many funds. Variance, a risk-sensitive measure, can only describe the uncertainty of earnings and does not accurately prove the size of the portfolio's losses. It is difficult to measure risk in a comprehensive and comprehensive manner and can only be used in specific financial instruments. VaR could be used in further research.

\section{REFERENCES}

[1] David. (2020, January 22). What Is An Investment Portfolio. Money Choice.

[2] Beattie, A. (2021, May 12). Understanding The History Of The Modern Portfolio. Investopedia.

[3] Ravipati, A. (2012). Markowitz's portfolio selection model and related problems (Doctoral dissertation, Rutgers University-Graduate School-New Brunswick).

[4] Kamal, J. B. (2012). Optimal portfolio selection in ex ante stock price bubble and furthermore bubble burst scenario from Dhaka stock exchange with relevance to Sharpe's single index model. Financial Assets and Investing, 3(3), 29-42.

[5] Kenton, W. (2021, May 7). S\&P 500 Index. Investopedia.

[6] Chen, J. (2021, May 7). Federal Funds Rate. Investopedia.

[7] Bilbao-Terol, A, Pérez-Gladish, B, \& Antomillbias, J. (2006). Selecting the optimum portfolio using fuzzy compromise programming and Sharpe's single-index model. Applied Mathematics and Computation, 182(1), 644-664.

[8] Putra, K. (2020). Study of Optimal Portfolio Performance Comparison: Single Index Model and Markowitz Model on LQ45 Stocks in Indonesia Stock Exchange. American Journal of Humanities and Social Sciences Research (AJHSSR), 237-244.

[9] Putra, K. (2020). Study of Optimal Portfolio Performance Comparison: Single Index Model and Markowitz Model on LQ45 Stocks in Indonesia Stock Exchange. American Journal of Humanities and Social Sciences Research (AJHSSR), 237-244. 